\title{
Photo-crosslinkable, insulating silk fibroin for bioelectronics with enhanced cell affinity
}

Jie Ju${ }^{1,2}$, Ning $\mathrm{Hu}^{1,3}$, Dana M. Cairns ${ }^{1}$, Haitao Liu ${ }^{1,4}$, Brian P. Timko ${ }^{1 *}$

1. Department of Biomedical Engineering, Tufts University, Medford, Massachusetts 02155, USA

2. Key Lab for Special Functional Materials, Ministry of Education, Henan University, Kaifeng, 475000 , China

3. State Key Laboratory of Optoelectronic Materials and Technologies, School of Electronics and Information Technology, Guangdong Province Key Laboratory of Display Material and Technology, Sun Yat-Sen University, Guangzhou, China

4. School of Materials Science and Technology, China University of Geosciences, Beijing 100083, China

* Corresponding author: Brian.Timko@tufts.edu 


\section{Abstract}

Bioelectronic scaffolds that support devices while promoting tissue integration could enable tissue hybrids with augmented electronic capabilities. Here, we demonstrate a Photo-crosslinkable Silk Fibroin (PSF) derivative and investigate its structural, electrical and chemical properties. Lithographically-defined PSF films offered tunable thickness and $<1 \mu \mathrm{m}$ spatial resolution, and could be released from a relief layer yielding freestanding scaffolds with cm-scale uniformity. These constructs were electrically insulating; multi-electrode arrays with PSF-passivated interconnects provided stable electrophysiological readouts from HL-1 cardiac model cells, brain slices and hearts. Compared to SU8, a ubiquitous biomaterial, PSF exhibited superior affinity toward neurons which we attribute to its favorable surface charge and enhanced attachment of poly-d-lysine adhesion factors. This finding is of significant importance in bioelectronics, where tight junctions between devices and cell membranes are necessary for electronic communication. Collectively, our findings are generalizable to a variety of geometries, devices and tissues, establishing PSF as a promising bioelectronic platform. 
Advances in bioelectronics promise to blur the line between living and artificial systems, enabling two-way communication between those disparate but complementary components ${ }^{1-3}$. Examples of bioelectronic devices include multi-electrode arrays (MEAs) and field-effect transistors (FETs) which have been interfaced with neuron or cardiac cells for multiplexed, realtime readouts of electrophysiological activity ${ }^{4}$, while other classes of devices have achieved ondemand drug delivery, localized stimulation, power generation and chemical sensing capabilities $^{5,6}$. Advances in nanoscience have played a key role in each of these areas by enabling cellular-scale, noninvasive devices such as transparent graphene $\mathrm{MEAs}^{7}$ and freestanding nanowire arrays ${ }^{8,9}$ that could access the cytosol for intracellular measurements.

One important challenge in bioelectronics - particularly with regards to 3D device configurations - relates to the device substrate, which must support devices and interconnects, provide adequate electrical passivation to prevent short circuits, and be biocompatible. Organ-level studies have been demonstrated using flexible, stretchable and bioresorbable materials ${ }^{10-12}$. More recently, highly flexible, macroporous mesh electronics were demonstrated ${ }^{13}$. Composed of devices and metallic interconnects sandwiched between layers of SU8, a photo-crosslinkable epoxy, mesh electronics functioned as device supports, electronic passivation, and tissue scaffold have been fabricated with tunable geometries using conventional photolithography processes. Significantly, they solved the problem of mechanical mismatches presented by conventional, rigid devices such as microwires and silicon-based microchips, thereby enabling seamless 3D device integration within engineered or primary tissues with minimal immune response. They have been implemented to realize synthetic nanoelectronic-innervated cardiac tissues ${ }^{14}$ and have also been injected into the brain ${ }^{15}$ or retina ${ }^{16}$ of live animal models to achieve chronically stable electrophysiological readouts. Collectively, these studies demonstrate the extraordinary potential for highly flexible electronics for achieving $3 \mathrm{D}$ bioelectronic interfaces with a wide variety of tissues.

By analogy to advances in tissue engineering ${ }^{17}$, future classes of bioelectronics should be tunable not only structurally but also chemically. One promising material for this purpose is silk fibroin, derived from the cocoon of Bombyx mori. Silk exhibits tunable mechanical properties ${ }^{18,19}$, excellent biocompatibility ${ }^{20}$ and programmable biodegradability ${ }^{21}$, making it an attractive material in tissue engineering. It also has potential for chemical customization through reactive amino acid side chain groups ${ }^{22}$. It has been demonstrated in a wide range of tissue models ${ }^{23}$, including those that incorporate co-cultures and functional innervation ${ }^{24}$. Bioelectronic devices containing silk as a major component would be complementary to these systems, potentially enhancing tissue/device integration.

Here, we describe a Photo-crosslinkable silk Fibroin (PSF) derivative and report for the first time how it may be used as a passivation material for bioelectronic devices. While photocrosslinkable forms of silk fibroin have been demonstrated previously ${ }^{25,26}$, their dielectronic properties in bioelectronic systems have not been explored, nor has their ability to adhere delicate cell lines such as human induced neural stem cells (hiNSCs) ${ }^{27}$ which have been integrated into 3D tissue cocultures and brain tissue models. We achieved PSF via an isocyanate-hydroxyl reaction and demonstrated a photoresist that was compatible with conventional photolithography. We found that thickness of the crosslinked PSF film could be tuned over an order of magnitude and after exposure and developing exhibited features with ca. $1 \mu \mathrm{m}$ resolution, rivaling the performance of SU8. Significantly, hiNSCs demonstrated remarkably improved adhesion to PSF compared to SU8, which we attribute to PSF's favorable surface charge and enhanced attachment of poly-dlysine adhesion factors. This finding is of significant importance in bioelectronics, where tight 
junctions between devices and cell membranes are necessary for efficient electronic communication. These findings establish PSF as a promising bioelectronic platform which could be extended to freestanding $3 \mathrm{D}$ configurations that could be stably integrated into a wide variety of tissue models.

\section{Results}

Synthesis of PSF. Figure 1 outlines our synthetic route toward PSF. First, native silk fibroin was extracted from cocoons and freeze dried as described previously ${ }^{28}$. Photo-crosslinkable methacrylate groups were then grafted onto pendant hydroxyl groups on the fibroin backbone by reaction with 2-isocyanatoethyl methacrylate (IEM) in $60^{\circ} \mathrm{C}$ Dimethylsulfoxide (DMSO) with $1 \mathrm{M}$ lithium chloride and dibutylin diaurate catalyst under nitrogen atmosphere. The reaction product was thoroughly rinsed with a mixture of acetone and ethanol, dried and lyophilized to form a PSF powder, then dissolved in hexafluoroisopropanol (HFIP), to achieve a clear amber-colored solution. We confirmed successful engraftment of the methacrylate groups by ${ }^{1} \mathrm{H}$ NMR. While the PSF reserved most of the functional groups in native fibroin, additional chemical shifts in PSF spectra were ascribed to the IEM modification: $1.88 \mathrm{ppm}$ (methyl group), $5.7 \mathrm{ppm}$ and $6.09 \mathrm{ppm}$ (vinyl group), $4.97 \mathrm{ppm}$ (methylene bridge adjacent to O), and 3.27 and $3.35 \mathrm{ppm}$ (methylene bridge adjacent to N) (Figure S1).

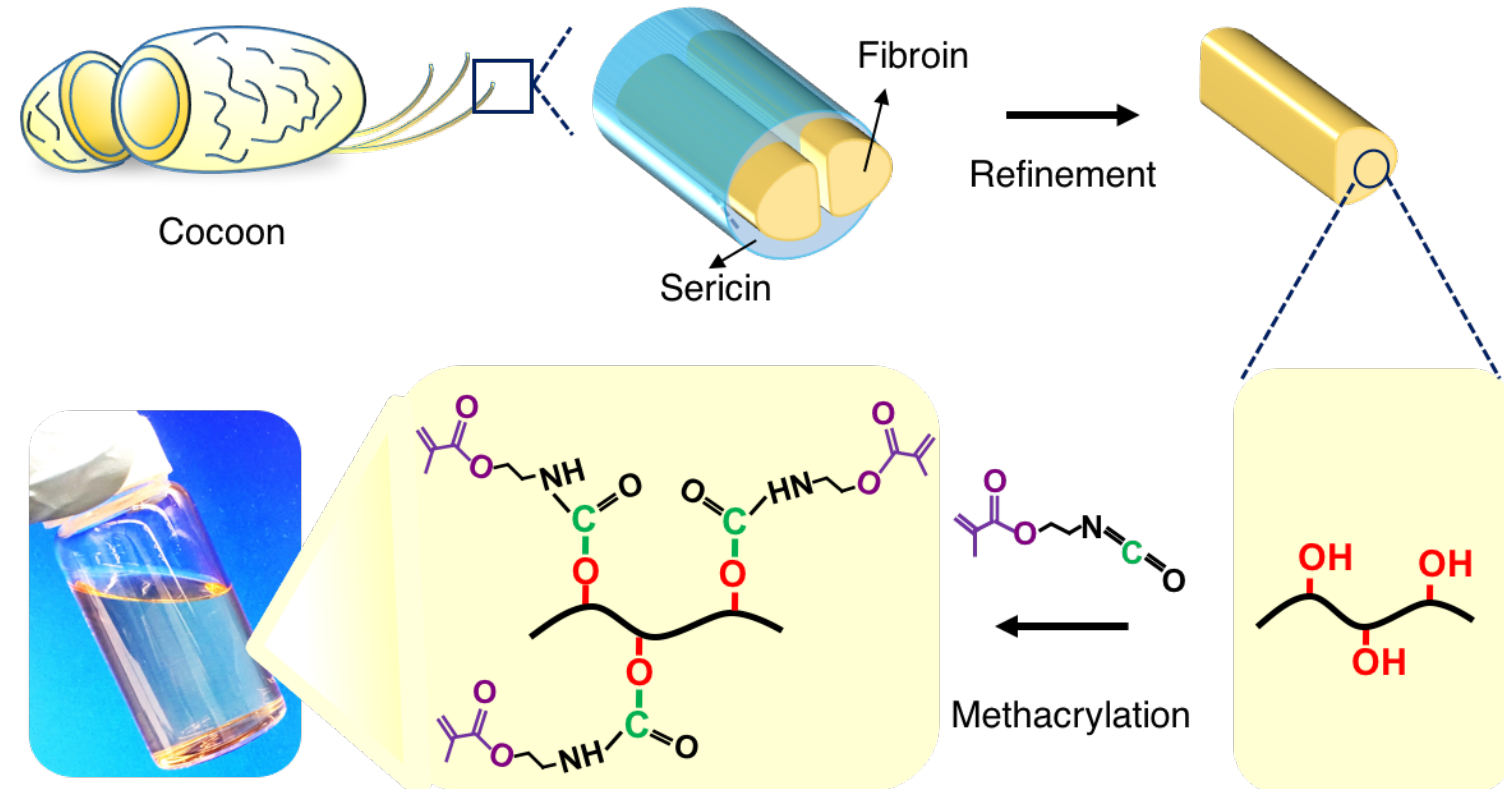

Figure 1. Synthetic route toward photo-crosslinkable fibroin (PSF). Native fibroin derived from cocoons was methacrylated through the hydroxyl groups on the chain to achieve PSF. After purification and freeze drying, PSF was readily dissolved in HFIP, yielding a clear amber-colored solution.

Characterization of lithographically-defined structures. After the synthesis of PSF, we assessed its feasibility as a photoresist (Figure 2a). We chose Irgacure 2959 as the photoinitiator (PI) to trigger the crosslinking of PSF under UV since Irgacure 2959 is a high efficacy PI and exhibits minimal toxicity to cells (PI refers to Irgacure 2959 in the following text other than special note) ${ }^{29,30}$. We prepared solutions of PSF and PI in HFIP at concentrations of $4 \%, 6 \%, 8 \%$, and $10 \%$ and $0.4 \%(\mathrm{w} / \mathrm{v})$, then spin-coated them on silicon substrates. After pre-baking the substrates at 
$80^{\circ} \mathrm{C}$ for $10-15 \mathrm{~min}$ and selectively crosslinking the PSF by UV photolithography, we post-baked the substrates for another $10 \mathrm{~min}$ at $80^{\circ} \mathrm{C}$ and then immersed them into a $\mathrm{LiCl} / \mathrm{DMSO}$ solution (1M) for 0.5 to $2 \mathrm{~min}$ to remove the unreacted PSF. Thermogravimetric analysis (TGA) and differential scanning calorimetry (DSC) both revealed that the glass transition of PSF was in a broad range, from about $110^{\circ} \mathrm{C}$ to over $170^{\circ} \mathrm{C}$, substantially higher than our pre-and post-bake temperatures (Figure S2).

We next assessed the suitability of PSF for achieving microscale structures by photolithography. First, we created a Tufts University logo pattern in which minimum feature sizes of ca. $1 \mu \mathrm{m}$ were clearly resolved (Figure $2 \mathrm{~b}-\mathrm{d}$ ). Next, we demonstrated crossbar motifs similar to those achieved with mesh electronics ${ }^{13}$. Figure $2 \mathrm{e}$ depicts the cross-sectional profile of the crossbars along the dashed red line, highlighting the sharp edges of the pattern. Significantly, height of the pattern (thickness of PSF film) could be controlled by varying the concentration of PSF in HFIP solution (Figure 2f) as well as the spin coating speed (Figure 2g), allowing us to tune the thickness between ca. $200 \mathrm{~nm}$ to $2500 \mathrm{~nm}$. We found that PSF could be resolved over a very broad exposure window, which makes the processing time more flexible. For example, films formed from 6\% PSF solution and $2000 \mathrm{rpm}$ spin rate exposed at doses between $1000 \mathrm{~mJ} / \mathrm{cm}^{2}$ to $12000 \mathrm{~mJ} / \mathrm{cm}^{2}$ yielded similar high-quality patterns after development (Figure S3). In addition, the as-prepared PSF patterns were stable against common solvents frequently used in microfabrication and cell culture including isopropyl alcohol (IPA), ethanol, methanol, dimethyl sulfoxide (DMSO), cell culture medium, and phosphate-buffered saline (PBS) for at least one month (Figure S4). Finally, we demonstrated that our silk films could be released from an underling relief layer to achieve highly-flexible, free-standing constructs that are amenable to macroporous, conformable electronics with device arrangements in 3D configurations (Figure $2 \mathrm{~g}$ ). Collectively, these features demonstrated the suitability of PSF for bioelectronic applications in which stable, high resolution structures and flexible conformations, including those in 3D, would be desirable.

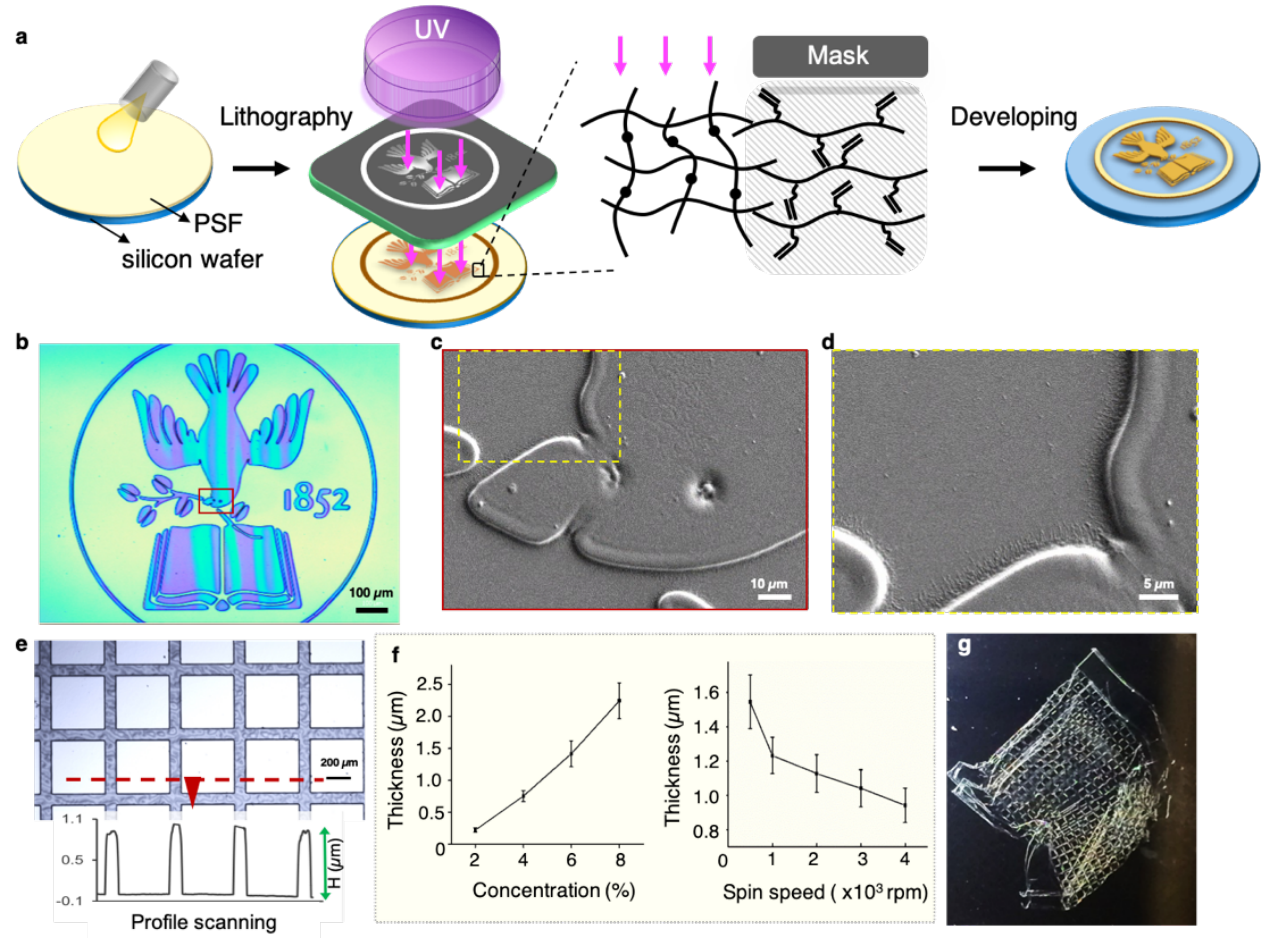


Figure 2. Microfabrication with PSF. a, Fabrication scheme using PSF as a negative photoresist, whereby an arbitrary pattern (e.g., Tufts logo) may be achieved by selective crosslinking of the PSF by conventional lithography followed by a developing step to wash away the unreacted material. b, Optical image of a PSF Tufts logo pattern and c-d, SEM expansions of regions in dotted boxes. e, Optical image of PSF crossbar pattern on glass substrate. The insert curve represents the height profile of structures shown in red dashed line. f, PSF film thickness as a function of PSF photoresist solution concentration $(\mathrm{N}=15)$ or spin speed during spin coating $(\mathrm{N}=4)$. g, Photo of a highly flexible, free-standing PSF construct achieved through etching underlying zinc relief layer.

Bioelectronic interfaces with silk-based MEAs. We next explored the feasibility of our silkbased structures to achieve stable bioelectronic interfaces with cell culture medium and living cells. Since bioelectronic devices are typically addressed by passivated interconnects, we first assessed the dielectric properties of PSF by electrical impedance spectroscopy (EIS). Gold working electrodes coated with dielectric films were submerged in PBS to mimic the conditions of a typical bioelectronic measurement. We then measured the impedance spectra across those films using a typical three-electrode setup consisting of a Pt wire counter electrode and $\mathrm{Ag} / \mathrm{AgCl}$ reference electrode (Fig. 3a). Films cast from 2\% PSF demonstrated poor passivation, potentially because of pinholes in the films which were evident under SEM (data not shown). In contrast, films cast from $4 \%, 6 \%$ and $8 \%$ PSF exhibited excellent dielectric properties over the range 10 $10^{6} \mathrm{~Hz}$, with corresponding impedance amplitude ratio $\left(\left|\mathrm{Z}_{\mathrm{PSF}, \mathrm{c}}\right| /\left|\mathrm{Z}_{\text {bare_Au }}\right|\right)$ of 14.6, 17.2 and 16.6 at $10^{3} \mathrm{~Hz}$, for $\mathrm{c}=4,6$ and $8 \%$ PSF, respectively. Significantly, these PSF films exhibited better insulating properties than the typically used dielectric layer of sputtered $200 \mathrm{~nm} \mathrm{SiO} 2$ films, which exhibited a ratio of only 2.79 times at $10^{3} \mathrm{~Hz}$.

We next assessed the ability of silk-passivated devices to form bioelectronic interfaces with living cells or tissue. Our devices consisted of MEAs with circular 30- $\mu$ m diameter Au recording elements whose interconnects were passivated by 6\% PSF films (Fig. 3b-d). Figure 3d is model of our device built on a printed circuit board (PCB) with PDMS chamber adhered to enable cell culturing. We initially assessed our devices with HL-1 cardiac muscle models ${ }^{31}$, which remained viable on our MEA substrates for at least 8 days in vitro (DIV). Figure 3e shows a false-colored SEM image of the HL-1 monolayer at 4 DIV, highlighting the cell (pink), recording element (light yellow) and silk passivation layer (yellow). Throughout the culture period HL-1 monolayers beat spontaneously with stable firing intervals of $0.27 \pm 0.01 \mathrm{~s}, 0.29 \pm 0.02 \mathrm{~s}$, and $0.26 \pm 0.01 \mathrm{~s}$ at 4,6 and 8 $\mathrm{DIV}$, respectively $(\mathrm{N}=3)$. Figure $3 \mathrm{f}$ and $3 \mathrm{~g}$ show extracellular recordings from a representative recording element at each of those time points, showing an increase in magnitude over time -48.2 $\pm 0.9 \mu \mathrm{V}, 218.3 \pm 0.5 \mu \mathrm{V}$, and $441.8 \pm 0.6 \mu \mathrm{V}$ at 4,6 and $8 \mathrm{DIV}$ respectively - consistent with an expected increase in cell-cell junctions and electrical synchrony throughout the culture over time (Figure S5). Significantly, the filtered baseline noise was consistently less than $4 \mu \mathrm{V}$ across all time points, and the strongest bioelectronic interfaces demonstrated signal-to-noise $>78$.

Our route toward silk-passivated bioelectronics represents a general platform that is readily extendable to a wide variety of applications. We anticipate that our devices will enable bioelectronic interfaces with a wide variety of cell or tissue types; to demonstrate this principle we achieved bioelectronic interfaces with ex vivo mouse brain slices and heart. Female Balb/c mice were sacrificed at age of week 6 , and the isolated hearts and brains were immediately interfaced to silk-passivated MEA devices to record signals with signal-to-noise of 48 and 9.2, respectively. (Fig. S6). In addition, we note that our silk films are highly transparent, with $>90 \%$ transmission 
at visible wavelengths. This transmissivity is similar to SU8 films ${ }^{32,33}$ as well as silk films that were not photo-crosslinked ${ }^{11}$, and opens avenues for simultaneous imaging and recording (Figure S7).
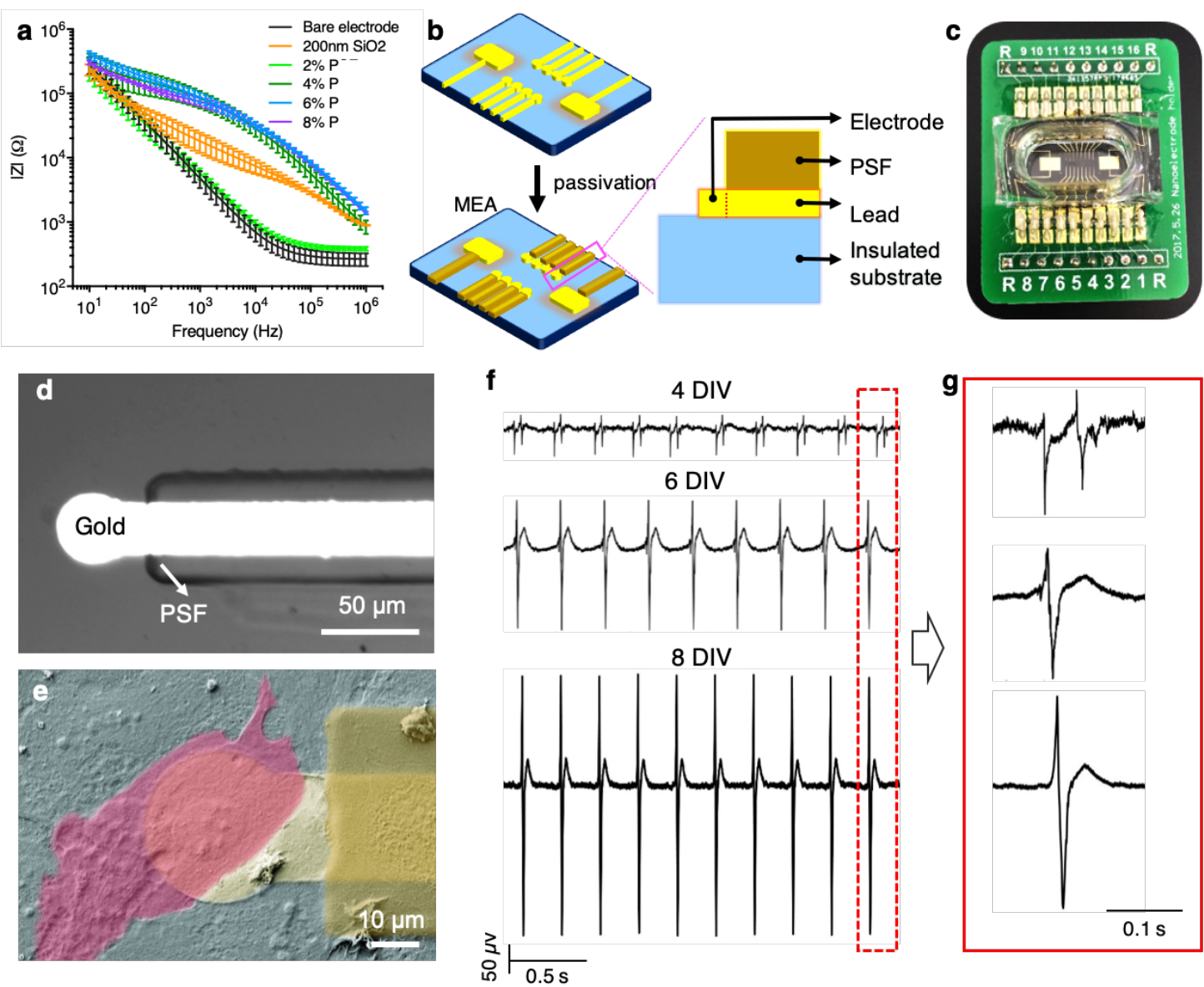

Figure 3. Bioelectronic measurements with PSF-based devices. a, Electrical impedance spectrum of PSF films spin spin-coated from 2-8\% (w/w) HFIP solutions, compared to bare gold electrodes and electrodes coated with a silicon dioxide layer with thickness of $200 \mathrm{~nm}(\mathrm{~N}=16)$. $\mathbf{b}$, Schematics of the MEA with interconnects passivated by PSFP. The expansion illustrates a side view of a single device element and passivated interconnect. c, Photograph of bioelectronic chip assembly including MEA, PDMS well and PCB interface board. d, Optical image of a single device with interconnect passivated by PSF. e, False-colored SEM image of HL-1 cultured on the PSF passivated device highlighting (bright yellow) gold layer (deep yellow) PSF passivation layer and (pink) single HL-1 cell. f, Extracellular signal from a spontaneously-beating HL-1 cell monolayer recorded on days 4, 6 and 8. g, Magnified signals in the red dashed box in e.

Characterization of cell adhesion. A crucial feature of bioelectronic scaffolds is that they must exhibit appropriate chemical properties to form tight, seamless interfaces with the surrounding cells. We studied this criterion using hiNSCs, which spontaneously differentiated in to neurons and have shown great potential in engineered brain and other innervated tissue models ${ }^{27,34}$. For the purposes of these studies we examined 2D systems, which enabled quantitative studies of cellular adhesion, migration and surface chemistry. 

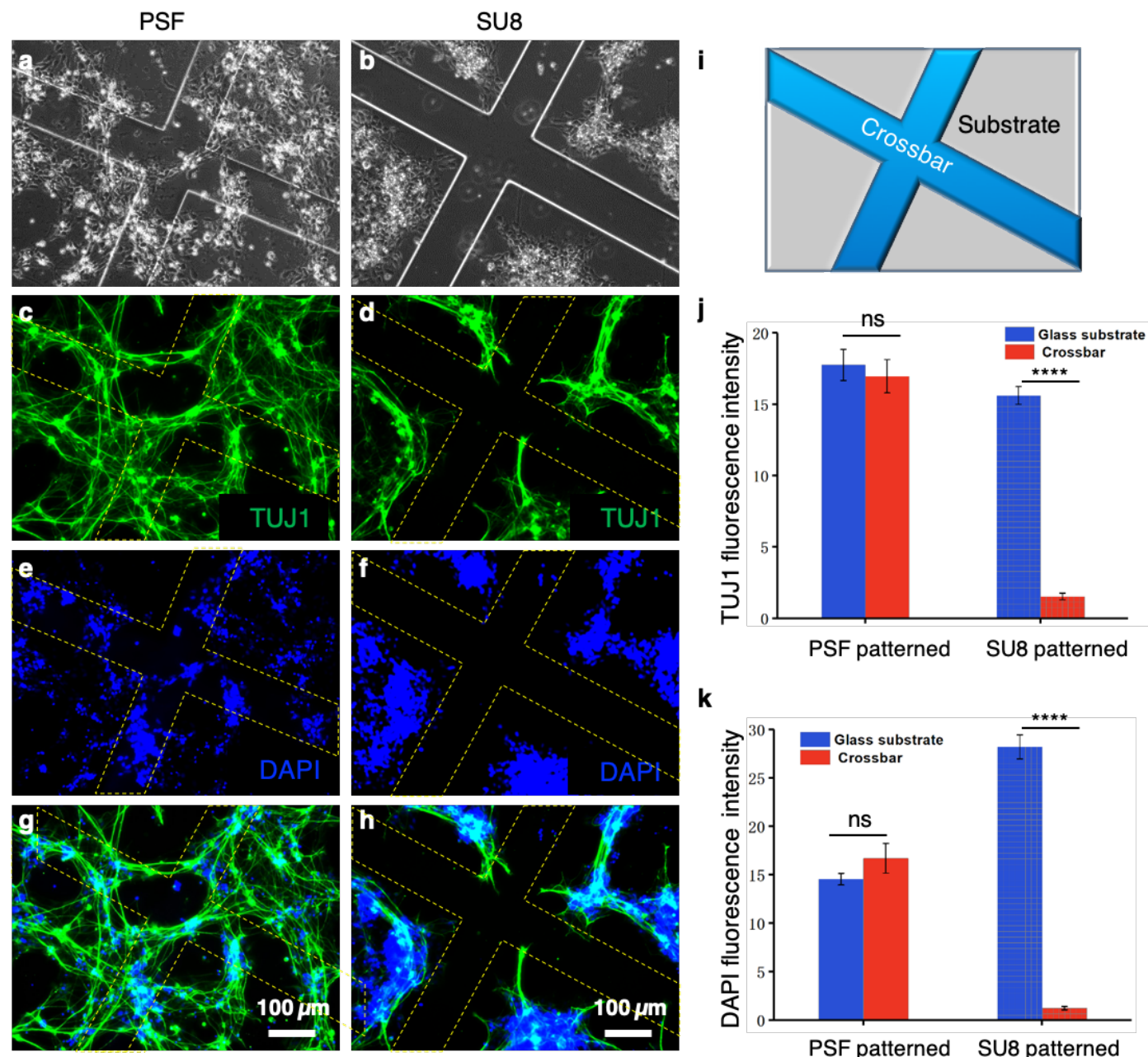

k

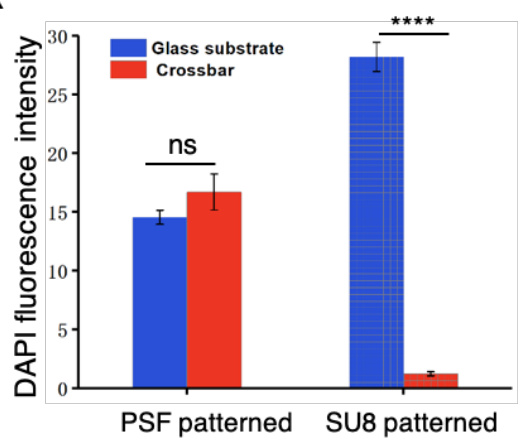

Figure 4. hiNSC adhesion study on microfabricated PSF and SU8 structures. a-b, Bright field microscope images of cells plated on (left) PSF and (right) SU8 patterned surfaces. The cells were stained with neuron specific marker antibody TUJ1 (c and d) and DAPI (e and f), with $\mathbf{g}$ and $\mathbf{h}$ merged images for both surfaces. $\mathbf{i}$, schematics of the patterned structure. $\mathbf{j}-\mathbf{k}$, Statistical analysis of varied fluorescence intensity for TUJ1 and DAPI on PSF and SU8 patterned surfaces, showing that cells distributing evenly on the PSF patterned surfaces, while avoiding SU8 framework (N=7).

We plated hiNSCs on the PSF-patterned glass wafer surfaces and maintained their growth in culture medium (see Methods). SU8-patterned glass wafers and pristine glass wafers were also seeded with hiNSCs at the same density as contrast and control groups. Immediately prior to plating the cells, all the surfaces were coated with poly-D-lysine (PDL) to promote hiNSC adhesion. After 5 days of culturing, the cells were fixed and stained with the neuron-specific marker $\beta$-III tubulin (TUJ-1) antibody and DAPI. Figure $4 \mathrm{a}$ and $4 \mathrm{~b}$ are the bright field images of hiNSCs on the PSF (a) and SU8 (b) patterned glass substrate. Figure 4c (4e) and 4d (4f) are corresponding fluorescence images stained with TUJ1 antibody (DAPI). Figure $4 \mathrm{~g}$ and $4 \mathrm{~h}$ are the merged fluorescent images. The yellow dashed lines outline edges of the PSF and SU8 crossbars (Figure 4i) to aid visualization. All of these images clearly show that hiNSCs distributed differently on the two types of surfaces: while hiNSCs appear both on the PSF crossbars and the interspaced glass substrates on the PSF patterned surfaces, they mostly avoid contacting with the SU8 crossbars on the patterned surfaces. Figure $4 \mathrm{j}$ and $4 \mathrm{k}$ are statistical analysis of the fluorescence 
intensity of the different regions on the PSF and SU8 patterned surfaces. This quantitative comparison demonstrates that the fluorescence intensity on the PSF crossbars was similar to that on the glass substrate irrespective of statistics based on TUJ-1 staining (Figure 4j) or DAPI staining (Figure 4k). However, the fluorescence intensity on the SU8 crossbars was much lower than that on the interspaced glass substrates, indicating fewer hiNSCs adhered to the SU8 crossbars. This distribution difference was even more apparent in the wide-field imaging, as shown from the bright field and fluorescent images in Figure S8, where the pristine glass wafer substrate was used as the control surface.

Since hiNSCs were plated on all surfaces at the same density and cultured under identical conditions, we hypothesized that the final distribution behavior arose from differential cell affinity to SU8 vs. PSF surfaces. To address this point we assessed the wettability of uniform PSF and SU8 films both before and after the PDL coating. These films were prepared in a fashion identical to the crossbar arrays presented in Figs. 4 and S8, except that they were uniformly crosslinked by excluding the photomask during exposure. Before coating, the PSF and SU8 film showed water contact angles (WCA) of $48.1 \pm 2.9^{\circ}$ and $89.0 \pm 2.3^{\circ}$, respectively (Figure $5 \mathrm{a}$ and $5 \mathrm{~b}$ ). After PDL coting at room temperature overnight, the WCA on the PSF film has increased to $61.7 \pm 1.5^{\circ}$, a change of approximate $13.6^{\circ}$ although still in the hydrophilic domain, indicating successful coating (Figure 5c) $)^{35}$; while the WCA on the SU8 decreased a little, to $86.5 \pm 1.8^{\circ}$, kept more hydrophobic than the post-coated PSF film (Figure 5d). ( $\mathrm{N}=5$ for all WCA measurements.) Since hydrophobicity is adverse to cell adhesion and moderate hydrophilicity promotes cell adhesion, this difference in the wettability on both surfaces in turn affects cell distribution ${ }^{36}$. 

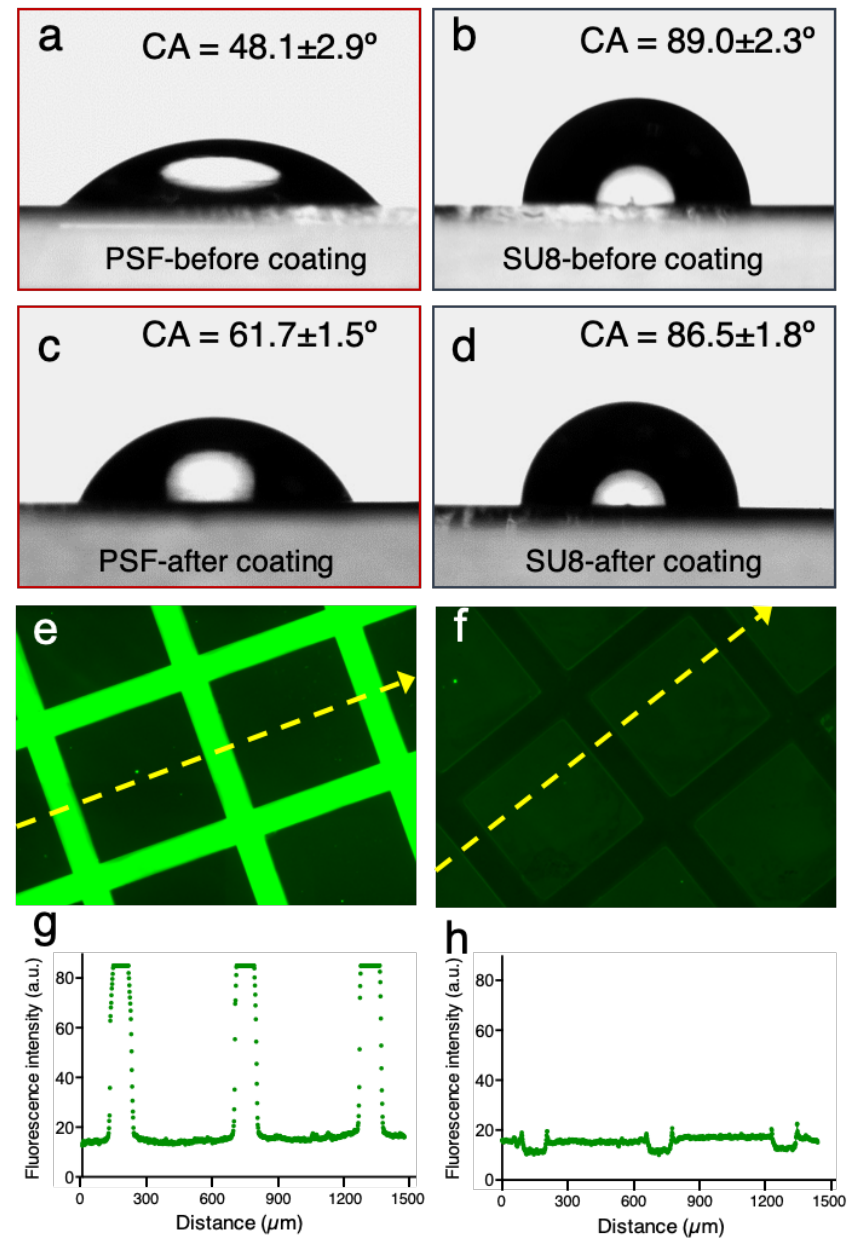

Figure 5. a-d, Contact angle measurements on PSF $(a, c)$ or SU8 $(b, d)$ films either with or without PDL coating. e-f, Fluorescence microscopy of FITC-PLL coated glass surfaces patterned with PSF or SU8 crossbars. g-h, Profile of the fluorescence intensity along the dashed yellow line in PSF and SU8 patterned glass substrate, respectively.

To visualize the differential coating effect on PSF and SU8, we used FITC-labelled poly-Llysine (PLL) to coat the PSF and SU8 patterned surfaces and imaged them under a fluorescent microscope. FITC-labelled PLL had the same coating mechanism as PDL on the substrate (physical absorption) but was also fluorescent ${ }^{37}$. Figure $5 \mathrm{e}$ and $5 \mathrm{f}$ are the PSF and SU8 patterned surfaces captured under the same exposing time. The PSF crossbars are brighter than the interspaced glass substrate, which is further brighter than the SU8 crossbars. Figure $5 \mathrm{~g}$ and $5 \mathrm{~h}$ are the fluorescence intensity profile along the yellow dashed line in Figure 5e and 5f. For both surfaces, the interspaced glass substrates keep the same value of around 16, but the PSF crossbars have a much higher value of nearly 90 and SU8 crossbars have a lower value of around 10. The different coating effect of PLL on the PSF, glass wafer, and SU8 surfaces can also be found in Figure S9 in wide-field imaging. All of these results confirmed that PLL was physically absorbed more on the PSF surface comparing with that on the SU8, which has leaded the different final cell distributing behavior. The probable reason for the different physical absorption is the varied surface electrical properties: PSF shows large negative charges in neutral solution, glass surface is less negative charged, and SU8 is more prone to be neutral ${ }^{38}$. When those surfaces are immersed 
in the positively charged PLL or PDL solution, the electrostatic interaction is strongest on the PSF surfaces, leading to being coated most and attracting the most cells, vice versa for the situation of SU8 surfaces.

\section{Discussion}

In this work we have investigated PSF, a photocrosslinkable derivative of silk fibroin which we achieved a photoresist and used to develop silk-based bioelectronic devices. While similar materials have been investigated previously, to the best of our knowledge this is the first study to specifically assess the unique properties of photocrosslinkable silk as they relate to bioelectronics. We demonstrated that PSF-based photoresists, compatible with standard photolithography, could achieve patterned features with cm-scale uniformity and feature sizes with critical length $<1 \mu \mathrm{m}$. This feature is important since it opens avenues for a wide variety of bioelectronic devices, ranging from short channel-length field-effect transistors (FETs) to organ-level structures, including bioelectronics-embedded brain or cardiac tissues. Moreover, we showed that our PSF exhibited not only promising structural properties but also excellent electrical insulating properties, which demonstrate that the material may be used both to support devices and also passivate electrical interconnects. This is a crucial feature for a wide range of bioelectronic devices, ranging from active FETs to passive MEAs ${ }^{4}$. As proof-of-principle and to demonstrate the robustness of our technique, we demonstrated that MEAs with silk-passivated interconnects could record signals both from cultured cells (HL-1) as well as primary tissues (brain, heart). Significantly, in the case of HL-1 cells we recorded signals with signal-to-noise $>78$, which compares favorably other devices recently used to study cardiac monolayers, including graphene MEAs with SU8 passivation ${ }^{7}$.

A significant contribution of this study - and perhaps the one that most distinguishes it from recent works involving SU8 - is our observation that PSF exhibited substantially improved properties for cell culture: it was more hydrophilic in its as-prepared state, more effectively bound the poly-d-lysine adhesion factor, and at least in the case of hiNSC-derived neurons far more effectively promoted soma adhesion as well as neurite extension. While highly flexible SU8 scaffolds have demonstrated remarkable promise in bioelectronics, providing stable recordings from brain or retina with minimal immune response, these studies have been largely confined to mature cells in vivo. The few demonstrations of SU8 in engineered tissue systems required a chemically-distinct matrix (e.g. coated poly(lactic-co-glycolic acid) fibers) to promote 3D adhesion and spreading. The ability of PSF to promote adhesion is likely to improve coupling between cell membranes and devices, thereby improving signal-to-noise ${ }^{39}$. This property is especially apropos within the context of nerve tissue engineering where neurons are relatively sparse compared to cardiac tissue. Since 3D models for brain and other innervated tissues have been achieved in silk scaffolds ${ }^{23,24}$, freestanding PSF-based bioelectronic scaffolds similar to the proof-of-concept shown in Fig. $2 \mathrm{~g}$ represent a logical route toward stable bioelectronics-embedded hybrids.

PSF represents a general platform that could be applied to a wide variety of cell and tissue types. The functionality of PSF described here could be further extended through covalentlyattached ligands, e.g. to achieve integrin-specific binding, direct neurite outgrowths, or achieve sustained drug release capabilities ${ }^{40}$. Moreover, we note that silk fibroin bioinks are compatible with $3 \mathrm{D}$ printing processes ${ }^{41}$, which in conjunction with techniques to print flexible and stretchable circuits $^{42}$ could enable complex bioelectronic scaffolds with geometry tailored to the specific application. Taken together, the advantages of PSF could enable entirely new classes of hybrid 
tissues with seamlessly integrated bioelectronics that monitor, modulate or otherwise augment tissue function.

\section{Methods}

\section{Refinement of silk fibroin}

Cocoons from B. Mori silkworm were cut into small pieces and placed into a boiling $0.02 \mathrm{M}$ $\mathrm{Na}_{2} \mathrm{CO}_{3}$ aqueous solution $30 \mathrm{~min}$. The flocculent fibroin in the solution was collected and rinsed with copious amount of DI water to remove any remaining $\mathrm{Na}_{2} \mathrm{CO}_{3}$. Afterwards, the fibroin was dried in an air convection fume hood thoroughly and then dissolved in $9.3 \mathrm{M} \mathrm{LiBr}$ aqueous solution in a $60^{\circ} \mathrm{C}$ oven for 2-4 hours. The as prepared fibroin solution was then dialyzed for 2 days using a dialysis membrane with molecular weight cutoff of $3 \mathrm{kDa}$. Particulates were removed by two centrifugation cycles at $9000 \mathrm{rpm}$ at $4^{\circ} \mathrm{C}$ for $20 \mathrm{~min}$. The supernatant was collected and lyophilized to achieve purified native silk fibroin.

\section{Synthesis of photo-crosslinkable silk fibroin}

$1.6 \mathrm{~g}$ lyophilized silk fibroin was dried in a $60^{\circ} \mathrm{C}$ oven overnight prior to further use and then dissolved in $160 \mathrm{ml} 1 \mathrm{M} \mathrm{LiCl} / \mathrm{DMSO}$ solution at a final concentration of $1 \%(\mathrm{w} / \mathrm{v})$. The mixture was maintained at $60^{\circ} \mathrm{C}$ with continuous stirring and degassing via nitrogen purge. After the solution became clear (ca. $20 \mathrm{~min}$ ), $3.18 \mathrm{ml}$ IEM and $60 \mathrm{ul}$ DBTDL were added into the solution quickly. The reaction was allowed to proceed for 5 hours before pouring into a mixture of cold acetone and ethanol (volume ratio of 1:1) to precipitate the white flocculent post-modified fibroin. The product was rinsed $3 \mathrm{x}$ with acetone/ethanol mixture, and washed $2 \mathrm{x}$ with DI water, then lyophilized for 1-2 days, yielding dried and purified photo-crosslinkable fibroin (PSF).

\section{NMR}

Films of native and functionalized fibroin were dissolved in 1M LiCl/DMSO-d6 and $\mathrm{H}$ spectra for both samples were collected using a Bruker AVANCE III $500 \mathrm{MHz}$ NMR spectrometer.

\section{Thermogravimetric analysis (TGA)}

Before analysis, all samples were dried at $60^{\circ} \mathrm{C}$. TGA was scanned from $0^{\circ} \mathrm{C}$ to $900^{\circ} \mathrm{C}$, with a ramp speed of $20^{\circ} \mathrm{C}$ per minute. DSC was scanned in modulated mode from $30^{\circ} \mathrm{C}$ to $190^{\circ} \mathrm{C}$, with ramp speed of $10^{\circ} \mathrm{C}$ per minute and modulation $\pm 1.59^{\circ} \mathrm{C}$ every $60 \mathrm{~s}$.

\section{Photolithography}

PSF dissolved in HFIP $(8 \%, 12 \%, 16 \%$, or $20 \%$, w/v) was mixed with Irgacure 2959 photoinitiator $(0.8 \% \mathrm{w} / \mathrm{v})$ in equivalent volume prior to use. The homogeneous solution was spin-coated on a glass wafer or silicon wafer ( $500 \mathrm{rpm}$ for $10 \mathrm{~s}$, ramped to and held at $2000 \mathrm{rpm}$ for $40 \mathrm{~s}$ ) then prebaked at $80^{\circ} \mathrm{C}$ for $5-10$ minutes. The film was then crosslinked using a photoaligner UV source (OAI Model 204IR, $20 \mathrm{~mW} / \mathrm{cm}^{2}$ ) with exposure times ranging from 50s to 600s as described in the main text. Afterwards, the film was post-baked at $80^{\circ} \mathrm{C}$ for $5-10$ minutes, developed in $1 \mathrm{M}$ $\mathrm{LiCl} / \mathrm{DMSO}$ solution for 0.5 - 2 minutes, washed with isopropyl alcohol (IPA) and finally dried with compressed air. Thicknesses of the resulting PSF films were measured using a profilometer (Dektak XT, Bruker Company, Germany). Freestanding scaffolds were fabricated on a $200 \mathrm{~nm}$ thick $\mathrm{Zn}$ relief layer, released by etching in dilute $\mathrm{HNO}_{3}$ solution $(7-8 \% \mathrm{v} / \mathrm{v})$ for $10-15 \mathrm{~min}$, then 
washed with copious amount of DI water. MEAs were prepared on silicon substrates. Electrodes and interconnects were deposited using a multilayer photoresist structure consisting of $400 \mathrm{~nm}$

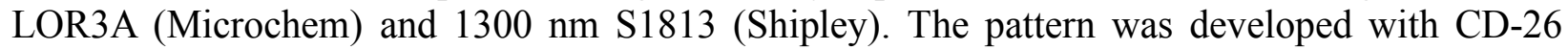
(Microchem), metallized with 10/40 nm Cr/Au (NSC 3000 DC Magnetron Sputter), and lifted off with Remover 1165 (Microchem). Interconnects were passivated with PSF in a second lithography step. All photomasks were custom designed in AutoCAD and prepared using a Heidelberg uPG501.

\section{Chip Assembly}

Chips were mounted onto custom-designed PCB boards (JLCPCB, Shenzhen, China). Electrical connections between the chip and PCB were formed with silver epoxy (CircuitWorks Conductive Epoxy). Poly(dimethylsiloxane) (PDMS) wells were cast as per manufacturer protocol (Sylgard $184)$ and adhered to oxygen plasma-treated chip surfaces (90 W, $180 \mathrm{~s})$ with a layer of PDMS oligomer that was then baked $\left(90-120 \mathrm{~min}\right.$ at $\left.100^{\circ} \mathrm{C}\right)$ to achieve a permanent bond. Finally, all exposed connections were insulated with a layer of epoxy glue (Gorilla).

\section{Electrical Impedance Spectroscopy (EIS)}

EIS spectra were collected with a Gamry Reference 600+ using a three-electrode setup consisting of a Au working electrode (surface area of approximate $706 \mu \mathrm{m}^{2}$ ), Pt wire counter electrode, and $\mathrm{Ag} / \mathrm{AgCl}$ reference electrode. Except for control group, all working electrodes were coated with a uniform dielectric layer: $\mathrm{SiO}_{2}$ deposited by sputter or PSF as described in the main text. All three electrodes were submerged in PBS and experiments were performed inside a Faraday cage. Impedance spectra were collected between $10^{6}$ and $10 \mathrm{~Hz}$ with an amplitude of $10 \mathrm{mV}$. The impedance amplitude ratio as calculated at $10^{3} \mathrm{~Hz}$ by comparing the impedance of $\mathrm{PSF}$ or $\mathrm{SiO}_{2}$ coated electrode system with that of the bare electrode system.

\section{hiNSC culturing and immunohistochemistry}

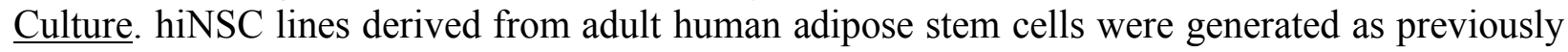
described. Surfaces were treated with $0.05 \mathrm{mg} / \mathrm{ml}$ PDL solution at RT overnight, then washed with PBS for 2-3 times. Cells (passage 16) were plated at $1.6 \times 10^{5}$ cells $/ \mathrm{cm}^{2}$ and the hiNSC maintaining medium (Knockout DMEM supplemented with 20\% knockout xeno-free SR, 1\% Glutamax, 1\% antibiotic-antimycotic and $0.1 \mathrm{mM} \beta$-mercapthanol) was changed once every three days.

Immunohistochemistry. Cells were fixed with $4 \%$ paraformaldehyde at $4^{\circ} \mathrm{C}$ for 15 min and then incubated with a blocking solution consisting of $6 \%$ goat serum and $0.1 \%$ TritonX-100 in PBS. The primary antibody (Rabbit TUJ-1) was added to the blocking buffer and incubated for $2 \mathrm{hr}$ at RT and then the sample was washed with PBS thoroughly. Cells were next incubated with the fluorescent secondary antibody (Abcam anti-rabbit IgG conjugated to Alexa 488) in blocking buffer for 1 hour and washed again with PBS. Nuclei were counterstained by incubating cells with DAPI solution for 10 minutes.

\section{HL-1 culturing and fixation for SEM}

Culture. HL-1 cells were plated at a density of $2 \times 10^{5}$ cells $/ \mathrm{cm}^{2}$ on the fibronectin pre-coated flask and MEA surfaces and maintained in the Claycomb medium supplemented with $10 \%$ fetal bovine serum, 2mM L-gultamine, $0.1 \mathrm{mM}$ norepinephrine, and $100 \mathrm{U} \mathrm{ml}^{-1}$ penicillin and $100 \mathrm{mg} / \mathrm{ml}$ streptomycin in a cell culturing incubator. Medium was changed daily.

SEM studies. HL-1 cells were fixed with $2 \%$ glutaraldehyde at $4^{\circ} \mathrm{C}$ for 1 hour then dehydrated 
with a gradient series of ethanol at $50 \%, 60 \%, 70 \%, 80 \%, 90 \%$ and $100 \%$ concentrations $(\mathrm{v} / \mathrm{v}), 10$ minutes for each concentration. Afterwards, the sample was dried using a liquid $\mathrm{CO}_{2}$ critical point dryer. Prior to SEM, samples were sputter coated with a $10 \mathrm{~nm}$ gold layer to impart conductivity.

\section{Mouse heart and brain signal recording}

Balb/c mice ( 6 week, female) were sacrificed by carbon dioxide asphyxiation. Their hearts and brains were carefully isolated from the surrounding tissue, then thoroughly washed with PBS (RT) to remove the blood. They were immediately interfaced MEA devices to record signals. All experiments were performed in accordance with US Animal Welfare Act and institutional guidelines and were approved by the Institutional Animal Care and Use Committee at Tufts University.

\section{Electrophysiology}

Extracellular signals were measured using a 16-channel data acquisition system (USB-ME16-FAISystem, Multichannel Systems, Germany) at a sampling frequency of $25 \mathrm{KHz}$. All signals were pre-amplified 1000x. They were filtered with a digital bandpass filter: $1 \mathrm{~Hz}$ to $2.25 \mathrm{kHz}$ for HL-1 cell measurements and $1 \mathrm{~Hz}$ to $1.25 \mathrm{kHz}$ for brain slice measurements.

\section{Surface wettability characterization}

Contact angles were evaluated using a custom-built measurement apparatus and calculated with OCA 20 software (DataPhysics). Each surface was measured at 5 different positions.

\section{Statistics}

Calculations were performed in GraphPad Prism or OriginLab. All the values are represented as means \pm standard error of the mean (SEM). Statistical analyses were performed using one-way analysis of variance (ANOVA), with $\mathrm{P} \leq 0.05$ considered statistically significant.

\section{Data availability}

The data supporting the findings of this work are available within the article and its Supplementary Information files. All other relevant data supporting the findings of this study are available from the corresponding author on request.

\section{Acknowledgements}

We thank Prof. David Kaplan for invaluable advice. We thank Dr. Will Collins, Dr. Tao Yang and Dr. Chengchen Guo for experimental assistance. We acknowledge a Tufts Collaborates grant to B.P.T., a Tufts Research Advancement Fund award to B.P.T. and a China Research Council Award, No. [2016]3100, to H.L. All authors thank the NIH for support through the Tissue Engineering Resource Center (P41 EB002520).

This work was performed in part at the Center for Nanoscale Systems (CNS), a member of the National Nanotechnology Coordinated Infrastructure Network (NNCI), which is supported by the National Science Foundation under NSF award no. 1541959. CNS is part of Harvard University. This work was also performed in part at the Tufts Micro and Nano Fabrication Facility with the assistance of Dr. James Vlahakis. 


\section{Author contributions}

J.J., N.H. and B.P.T. conceived the idea and designed the experiments. J.J., N.H., D.M.C. and H.L. performed the experiments. J.J., N.H. and H.L. collected and analysed the data. J.J. and B.P.T. wrote the text and all authors reviewed and revised. B.P.T. supervised the study.

\section{Additional information}

Supplementary information accompanies this paper.

Competing interests: The authors declare no competing interests.

Corresponding author: Brian P. Timko (Brian.Timko@tufts.edu)

\section{ORCID IDs}

J.J.: https://orcid.org/0000-0001-8931-3270

N.H.: https://orcid.org/0000-0001-7178-3952

D.M.C.: https://orcid.org/0000-0003-0574-8395

H.L.: https://orcid.org/0000-0003-1824-0304

B.P.T.: https://orcid.org/0000-0002-7958-8980

\section{References}

1 Zhang, A. \& Lieber, C. M. Nano-bioelectronics. Chem. Rev. 116, 215-257 (2016).

2 Liu, H. et al. Nanobiotechnology: 1D nanomaterial building blocks for cellular interfaces and hybrid tissues. Nano Res. 11, 5372-5399 (2018).

3 Luo, Z., Weiss, D. E., Liu, Q. \& Tian, B. Biomimetic approaches toward smart bio-hybrid systems. Nano Res. 11, 3009-3030 (2018).

4 Spira, M. E. \& Hai, A. Multi-electrode array technologies for neuroscience and cardiology. Nat. Nanotechnol. 8, 83-94 (2013).

5 Feiner, R. et al. Engineered hybrid cardiac patches with multifunctional electronics for online monitoring and regulation of tissue function. Nat. Mater. 15, 679-685 (2016).

6 Ding, W., Wang, A. C., Wu, C., Gao, H. \& Wang, Z. Human-machine interfacing enabled by triboelectric nanogenerators and tribotronics. Adv. Mater. 4, 1800487 (2019).

7 Rastogi, S. K. et al. Graphene microelectrode arrays for electrical and optical measurements of human stem cell-derived cardiomyocytes. Cell. Mole. Bioeng. 11, 407-418 (2018).

8 Tian, B. et al. Three-dimensional, flexible nanoscale field-effect transistors as localized bioprobes. Science 13, 830-834 (2010).

9 McGuire, A. F., Santoro, F. \& Cui, B. Interfacing cells with vertical nanoscale devices: applications and characterization. Annu. Rev. Anal. Chem. 11, 101-126 (2018).

10 Timko, B. P. et al. Electrical recording from hearts with flexible nanowire device arrays. Nano Lett. 9, 914-918 (2009).

11 Kim, D.-H. et al. Dissolvable films of silk fibroin for ultrathin conformal bio-integrated electronics. Nat. Mater. 9, 511-517 (2010).

12 Liu, Y., Pharr, M. \& Salvatore, G. A. Lab-on-skin: a review of flexible and stretchable electronics for wearable health monitoring. ACS Nano 11, 9614-9635 (2017).

13 Dai, X., Hong, G., Gao, T. \& Lieber, C. M. Mesh nanoelectronics: seamless integration of 
electronics with tissues. Acc. Chem. Res. 51, 309-318 (2018).

14 Dai, X., Zhou, W., Gao, T., Liu, J. \& Lieber, C. M. Three-dimensional mapping and regulation of action potential propagation in nanoelectronics-innervated tissues. Nat. Nanotechnol. 11, 776-782 (2016).

$15 \mathrm{Fu}, \mathrm{T}$-M. et al. Stable long-term chronic brain mapping at the single-neuron level. Nat. Methods 13, 875-882 (2016).

16 Hong, G. et al. A method for single-neuron chronic recording from the retina in awake mice. Science 360, 1447-1451 (2018).

17 Khademhosseini, A. \& Langer, R. A decade of progress in tissue engineering. Nat. Protoc. 11, 1775-1781 (2016).

18 Mitropoulos, A. N. et al. Transparent, nanostructured silk fibroin hydrogels with tunable mechanical properties. ACS Biomater. Sci. Eng. 1, 964-970 (2015).

19 Zhu, Z. et al. High-strength, durable all-silk fibroin hydrogels with versatile processability toward multifunctional applications. Adv. Funct. Mater. 28, 1704757 (2018).

20 Kapoor, S. \& Kundu, S. C. Silk protein-based hydrogels: Promising advanced materials for biomedical applications. Acta Biomater. 31, 17-32 (2016).

21 Brenckle, M. A. et al. Modulated Degradation of Transient Electronic Devices through Multilayer Silk Fibroin Pockets. ACS APPl. Mater. Interfaces 7, 19870-19875 (2015).

22 Murphy, A. R. \& Kaplan, D. L. Biomedical applications of chemically-modified silk fibroin. J. Mater. Chem. 19, 6443-6450 (2009).

23 Abbott, R. D., Kimmerling, E. P., Cairns, D. M. \& Kaplan, D. L. Silk as a biomaterial to support long-term three-dimensional tissue cultures. ACS APPl. Mater. Interfaces 8, 2186121868 (2016).

24 Wang, S. et al. In vitro 3D corneal tissue model with epithelium, stroma, and innervation. Biomaterials 112, 1-9 (2017).

25 Kurland, N. E., Dey, T., Kundu, S. C. \& Yadavalli, V. K. Precise patterning of silk microstructures using photolithography. Adv. Mater. 25, 6207-6212 (2013).

26 Pal, R. K. et al. Fabrication of precise shape-defined particles of silk proteins using photolithography. Eur. Polym. J. 85, 421-430 (2016).

27 Cairns, D. M. et al. Expandable and Rapidly Differentiating Human Induced Neural Stem Cell Lines for Multiple Tissue Engineering Applications. Stem Cell Rep. 7, 557-570 (2016).

28 Rockwood, D. N. et al. Materials fabrication from Bombyx mori silk fibroin. Nat. Protoc. 6, 1612-1631 (2011).

29 Mironi-Harpaz, I., Wang, D. Y., Venkatraman, S. \& Seliktar, D. Photopolymerization of cellencapsulating hydrogels: crosslinking efficiency versus cytotoxicity. Acta Biomater. 8, 18381848 (2012).

30 Williams, C. G., Malik, A. N., Kim, T. K., Manson, P. N. \& Elisseeff, J. H. Variable cytocompatibility of six cell lines with photoinitiators used for polymerizing hydrogels and cell encapsulation. Biomaterials 26, 1211-1218 (2005).

31 Claycomb, W. C. et al. HL-1 cells: A cardiac muscle cell line that contracts and retains phenotypic characteristics of the adult cardiomyocyte. Proc. Natl. Acad. Sci. U.S.A. 95, 29792984 (1998).

32 Vernekar, V. N. et al. SU-8 2000 rendered cytocompatible for neuronal bioMEMS applications. J. Biomed. Mater. Res. A 89, 138-151 (2009).

33 Zhang, Y. et al. Application of SU-8 as the Insulator toward a Novel Planar Microelectrode Array for Extracellular Neural Recording. Proc. 2010 5th IEEE NMES, 395-398 (2010). 
34 Gage, F. H. \& Temple, S. Neural stem cells: generating and regenerating the brain. Neuron 80, 588-601 (2013).

35 Moy, E., Lin, F. Y. L. H., Vogtle, J. W., Policova, Z. \& Neumann, A. W. Contact angle studies of the surface properties of covalently bonded poly-L-lysine to surfaces treated by glowdischarge. Colloid Polym. Sci. 272, 1245-1251 (1994).

36 Valamehr, B. et al. Hydrophobic surfaces for enhanced differentiation of embryonic stem cellderived embryoid bodies. Proc. Natl. Acad. Sci. U.S.A. 105, 14459-14464 (2008).

37 Kim, Y. H., Baek, N. S., Han, Y. H., Chung, M.-A. \& Jung, S.-D. Enhancement of neuronal cell adhesion by covalent binding of poly-D-lysine. J. Neurosci. Methods 202, 38-44 (2011).

38 Lammel, A. S., Hu, X., Park, S.-H., Kaplan, D. L. \& Scheibel, T. R. Controlling silk fibroin particle features for drug delivery. Biomaterials 31, 4583-4591 (2010).

39 Blau, A. Cell adhesion promotion strategies for signal transduction enhancement in microelectrode array in vitro electrophysiology: An introductory overview and critical discussion. Curr. Opin. Colloid Interface Sci. 18, 481-492 (2013).

40 Chen, J., Venkatesan, H. \& Hu, J. Chemically modified silk proteins. Adv. Eng. Mater. 20, 1700961 (2018).

41 Kim, S. H. et al. Precisely printable and biocompatible silk fibroin bioink for digital light processing 3D printing. Nat. Commun. 9, 1620 (2018).

42 Mohammed, M. G. \& Kramer, R. All-printed flexible and stretchable electronics. Adv. Mater. 29, 1604965 (2017). 